\title{
Over-the-scope clip to close a fistula after removing a percutaneous endoscopic gastrostomy tube
}

Percutaneous endoscopic gastrostomy (PEG) feeding is indicated in patients with increased nutritional requirements, such as patients with cystic fibrosis [1]. Refractory gastrocutaneous fistulas are a recognised complication following PEG tube removal. We describe the case of a patient with a nonhealing gastrocutaneous fistula that was successfully closed using an over-the-scope clip (OTSC).

Following PEG tube removal, a patient with cystic fibrosis had a persistent discharge and discomfort from a nonhealing gastrocutaneous fistula for 4 months (๑ Fig. 1).

An endoscopy was performed, and the gastric aspect of the fistula was successfully closed using three Resolution endoscopic clips (Boston Scientific, USA) (๑ Fig. 2).

After 2 weeks of symptomatic relief, the patient's symptoms recurred. Follow-up endoscopy revealed the absence of the initial clips, and so an alternative OTSC (Ovesco Endoscopy GmbH, Tübingen, Germany) was used ( Fig. 3).

The margins of the fistula were apposed with graspers, and the clip then deployed by using the external hand wheel, with the patient under conscious sedation with midazolam ( $\bullet$ Fig. 4 and 5 ).

Immediate closure of the fistula was confirmed by the inability to flush water through the tract into the stomach.

The patient was asymptomatic for 18 weeks, after which he developed further symptoms requiring elective surgical intervention.

Several methods have been described for closing nonhealing gastrocutaneous fistulas, including endoscopic clips, fibrin sealant, sutures, and a combination of these techniques [2,3]. Clip retention is a desirable property of endoscopic clips in this setting. Resolution clips have been reported to have significantly higher long-term retention than other commercially available clips [4], but despite their use in this patient, his symptoms recurred. However, wound healing was likely to be affected by the patient's underlying disease.

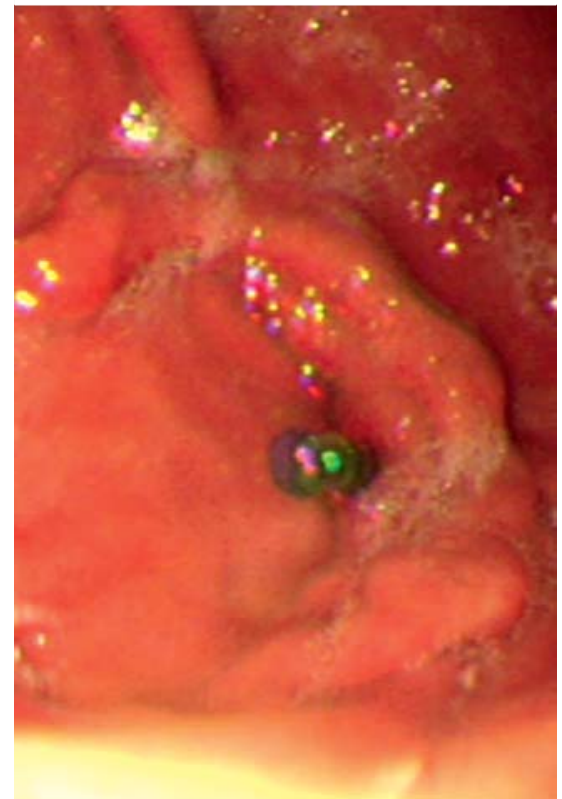

Fig. 1 Plastic catheter passed through the fistula tract into the stomach.

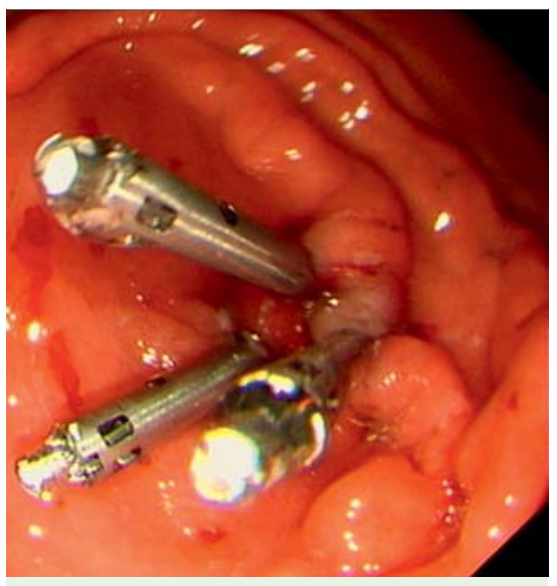

Fig. 2 Fistula closed with endoscopic clips.

The OTSC has been described for use in natural orifice transluminal endoscopic surgery [5]. OTSCs appear to be effective for closing acute perforations and fistulas in the short to medium term. This is the first report of the use of OTSCs to treat a nonhealing gastrocutaneous fistula following PEG removal.

Competing interests: None

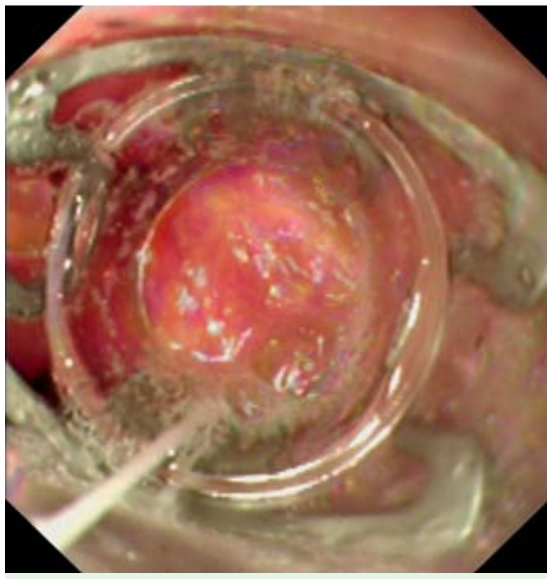

Fig. 3 Over-the-scope clip (OTSC) prior to clip deployment.

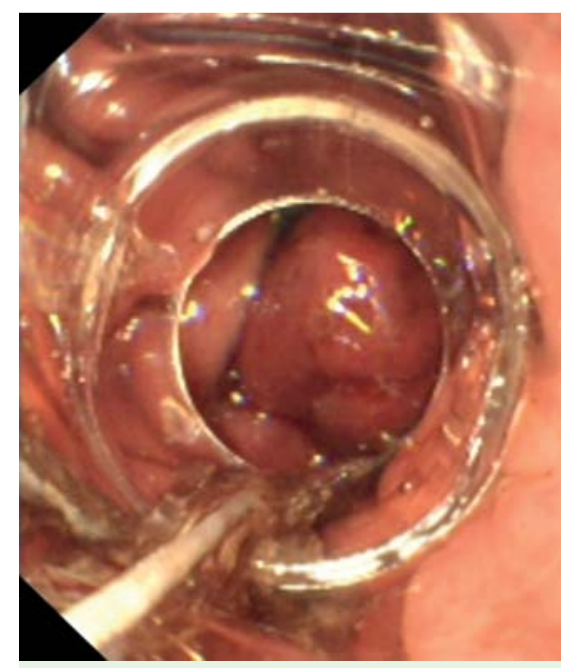

Fig. 4 Over-the-scope clip (OTSC) after clip deployment, with engorgement of the gastric mucosa.

Endoscopy_UCTN_Code_TTT_1AO_2AK

\section{J. K. Turner ${ }^{1}$, J. J. Hurley ${ }^{1}$, I. Ketchell ${ }^{2}$,}

\section{S. Dolwani ${ }^{1}$}

1 Department of Gastroenterology, University Hospital Llandough, Cardiff, UK

2 Department of Respiratory Medicine, University Hospital Llandough, Cardiff, UK 


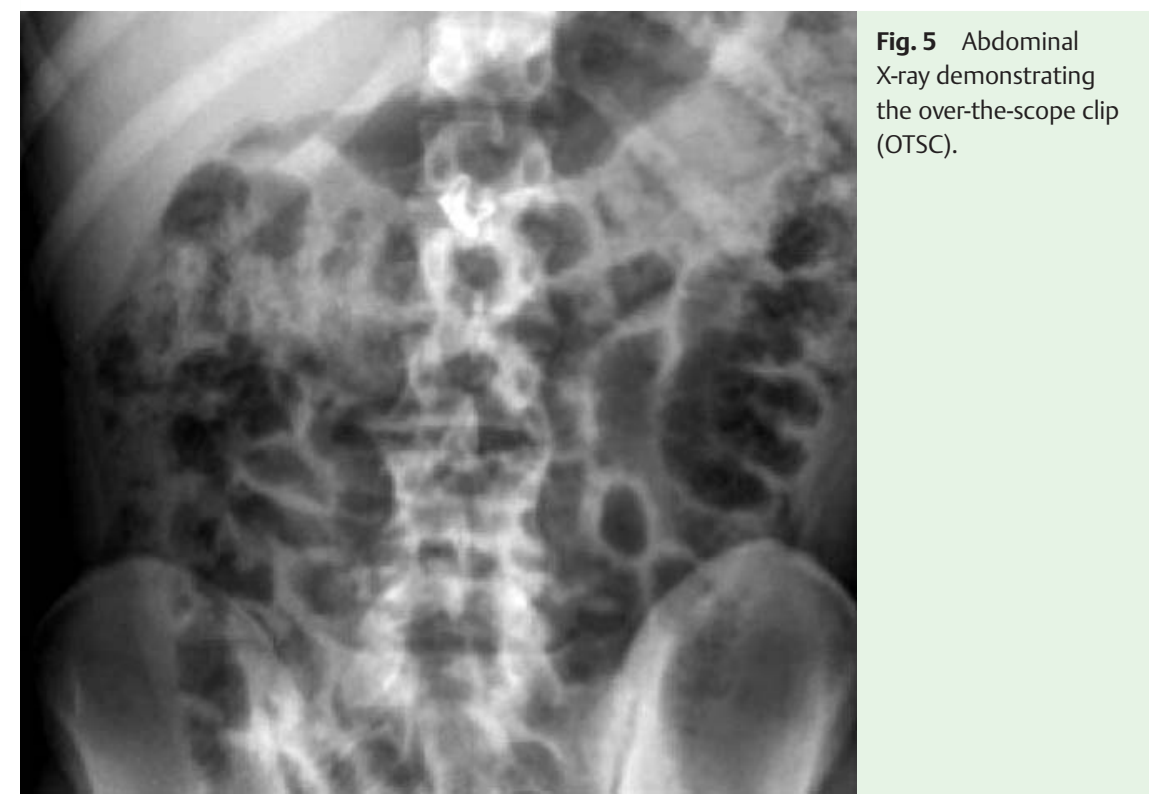

\section{References}

1 Stroud M, Duncan H, Nightingale J. British Society of Gastroenterology. Guidelines for enteral feeding in adult hospital patients. Gut 2003; 52: vii1 - vii12

2 Akhras J, Tobi M, Zagnoon A. Endoscopic fibrin sealant injection with application of hemostatic clips: a novel method of closing a refractory gastrocutaneous fistula. Dig Dis Sci 2005; 50: 1872 - 1874

3 Alberti-Flor JJ. Percutaneous-endoscopic suturing of gastrocutaneous fistula: report of 2 cases. Gastrointest Endosc 2002; 56: $751-753$

4 Shin EJ, Ko CW, Magno P et al. Comparative study of endoscopic clips: duration of attachment at the site of clip application. Gastrointest Endosc 2007; 66: 757-761

5 Schurr MO, Arezzo A, Ho CN et al. The OTSC clip for endoscopic organ closure in NOTES: device and technique. Minim Invasive Ther Allied Technol 2008; 17: 262 - 266

\section{Bibliography}

DOI $10.1055 / \mathrm{s}-0030-1255693$

Endoscopy 2010; 42: E197-E198

(c) Georg Thieme Verlag KG Stuttgart · New York . ISSN 0013-726X

\section{Corresponding author}

J. Turner

Department of Gastroenterology

University Hospital Llandough

Penlan Road

Cardiff

CF64 2XX

UK

Fax: +44-2920-715538

jkturner76@hotmail.com 